\title{
Configurational phases in elastic foams under lengthscale-free punching
}

\author{
Tapan Sabuwala ${ }^{\mathrm{a}, *}, \mathrm{Xiangyu}_{\mathrm{Dai}}{ }^{\mathrm{b}}$, Gustavo Gioia $^{\mathrm{a}}$ \\ a Okinawa Institute of Science and Technology Graduate University, Onna, Okinawa 904-0495, Japan \\ ${ }^{b}$ Department of Mechanical Science and Engineering, University of Illinois, Urbana, IL 61801, USA
}

\begin{abstract}
We carry out experiments with brick-like specimens of elastic open-cell (EOC) foams of three relative densities. Individual specimens may be "tall" (height $=$ width $=$ depth) or "short" ( 2 height $=$ width $=$ depth). We place each specimen on a supporting plate and use a lengthscale-free (wedge-shaped or conical) punch to apply forces downward along the specimen's height. Regardless of the type of specimen, the forcepenetration curves remain linear, for the wedge-shaped punch, or quadratic, for the conical punch, up to a sizable penetration commensurate with the smallest lengthscale of the specimen. After that there is an abrupt, all-but-discontinuous change in stiffness: if the specimen is tall, the stiffness drops; if the specimen is short, the stiffness shoots up. To analyze these curious experimental results, we posit that EOC foams can be found in either of two configurational phases, here termed the low-strain phase and the high-strain phase, which share a two-dimensional interface (a surface of strain discontinuity). The analysis may be outlined as follows. In the first part of an experiment, there obtains a "similarity regime" in which the penetration of the punch and the radius of the interface are the only prevailing lengtshcales (because the punch is lengthscale free). In this case, it is possible to show that the force-penetration curve must be linear, or quadratic, depending on whether the punch be wedge-shaped or conical, respectively. This prediction of the analysis is consistent with the experiments. In time, the similarity regime breaks down when the interface reaches one of the specimen's boundaries distal to the tip of the punch. If the specimen is tall, the soft, stress-free lateral boundary is reached first, and the stiffness must drop; if the specimen is short, the hard boundary in contact with the supporting plate is reached first, and the stiffness must shoot up. These predictions too are consistent with the experiments. To provide direct empirical evidence of the interface, we use a digital-image correlation method. Lastly, we run computational simulations of all the experiments, using finite elements and the skeleton-and-bubble model of EOC foams. The computational results are in good accord with the experimental ones, and they allow us to carry out a detailed validation of the analysis. Our findings evince the cardinal role of configurational phases in the mechanics of EOC foams.
\end{abstract}

Keywords: elastic foams, cellular material, phase transformation, foam experiments, indentation

\footnotetext{
${ }^{*}$ Corresponding author

Email address: tapan.sabuwala@oist.jp (Tapan Sabuwala)
} 


\section{Introduction}

An elastic open-cell (EOC) foam is a lightweight cellular material that may be roughly described as a three-dimensional, quasiperiodic network of elastic bars, or skeleton. Most EOC foams are transversely isotropic. The axis of transverse isotropy is the rise direction of the foam, a concept related to the foaming process by which EOC foams are commonly made.

EOC foams are commonly made by promoting the expansion of very many tiny, uniformly-distributed cavities within a heated sheet of polymer, typically polyether polyurethane. The cavities expand anisotropically, with a preferential bias for the direction normal to the sheet of polymer. This is the rise direction of the foam - the direction in which the sheet of polymer swells the most during the foaming process (Artavia and Macosko, 1994).

As the cavities continue to expand, the walls between adjacent cavities become thinner and thinner. In time, adjacent cavities link up between themselves through a set of holes, and each cavity ends up surrounded by a roughly prolate-spheroidal, cage-like (or "open") cell. The bars of the cage-like cells make up the skeleton.

The key geometrical parameter of an EOC foam is the relative density, which can be as low as 0.01. It can be defined as the ratio of the volume of the foam proper (skeleton only) to the volume of the foam (skeleton plus cavities). For an EOC foam of relative density 0.04 (a typical value), the skeleton occupies but $4 \%$ of the volume of the foam, and the remaining $96 \%$ is empty space.

EOC foams are "castles in the air" (Perkowitz, 2001): lightweight, soft materials that can be squeezed to a fraction of their original bulk by the application of modest compressive stresses. Such materials are well suited to take in much energy while curbing force buildup under pressure; for this reason, they are widely used in protective gear (helmets, knee pads), impact-resistant packages, sandwich panels, sports shoes, car seats, bedding, and upholstery. In these and other applications, EOC foams are routinely subjected to punching forces. And yet, research on the mechanical behaviour of EOC foams under punching has been limited in scope and largely restricted to experimental work. For the most part, punching experiments have been used to estimate a foam's "indentation resilience" (Chan and Evans, 1998) and elastic modulus (Yu et al., 2006), and to help validate constitutive models of foams (Mills and Gilchrist, 2000; Mills and Lyn, 2001; Lyn and Mills, 2001; Schrodt et al., 2005).

Our aim here is to carry out punching experiments that are designed to probe, with the aid of analysis and computations, the physics of microstructural deformation in EOC foams. Crucial to these experiments is the use of punches that lack an intrinsic lengthscale. 


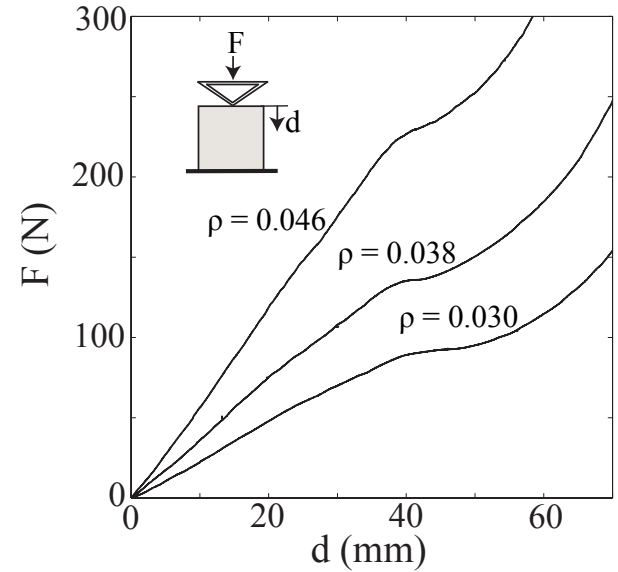

(a)

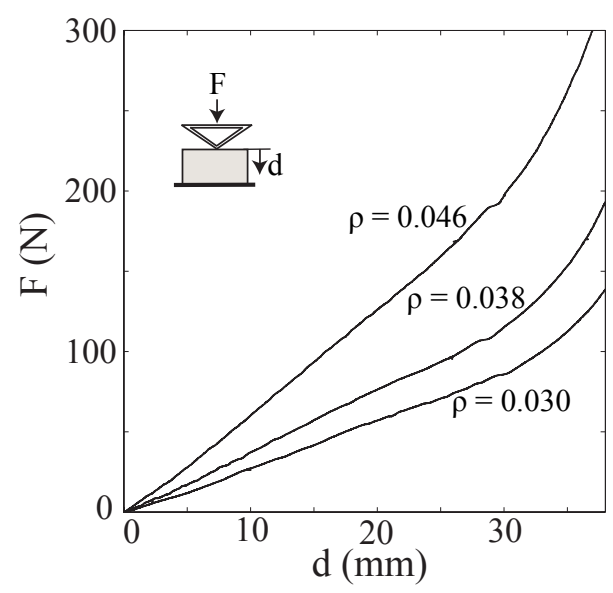

(b)

Figure 1: Force-penetration curves from experiments with the wedge-shaped punch (angle of attack $=90^{\circ}$ ). $\rho$ denotes the relative density of a foam. (a) Tall specimens. Inset: schematic of the experiments. (b) Short specimens. Inset: schematic of the experiments. Note that the force-penetration curves remain linear up to $d \approx 40 \mathrm{~mm}$, at which point there is either an abrupt falloff in stiffness (for the tall specimens) or an abrupt rise in stiffness (for the short specimens).

\section{Experiments}

We carry out experiments on a set of three polyether-polyurethane EOC foams of relative densities 0.030, 0.038 and 0.046 (Appendix A). We cut out two specimens of each foam: a tall specimen that is $10 \mathrm{~cm}$ in height, $10 \mathrm{~cm}$ in width, and $10 \mathrm{~cm}$ in depth; and a short specimen that is $5 \mathrm{~cm}$ in height, $10 \mathrm{~cm}$ in width, and $10 \mathrm{~cm}$ in depth. The height of either specimen is parallel to the rise direction of the foam.

We compress the specimens along the height, using either a wedge-shaped punch or a conical punch, and measure the punching force $F$ as a function of the penetration of the punch, $d$ (Appendix A). We show the force-penetration curves in Fig. 1 (experiments with the wedge-shaped punch) and in Fig. 2 (experiments with the conical punch).

The force-penetration curves display some striking features. For the wedge-shaped punch, the curves remain linear up to a penetration of some $40 \mathrm{~mm}$. For the conical punch, the curves remain quadratic up to a comparably large penetration. In either case, an all-but-discontinuous change in stiffness supervenes at a penetration of some $40 \mathrm{~mm}$. For the tall specimens, the stiffness drops abruptly and the force-penetration curves become (momentarily) almost level. For the short specimens, the stiffness shoots up and the forcepenetration curves enter a stage of accelerating hardening.

In the next section, we argue that these experimental results reveal the primary deformational mode of EOC foams under compression, whereby changes in average strain are accommodated by means of suitable motions of the two-dimensional interface between the configurational phases of a foam. 


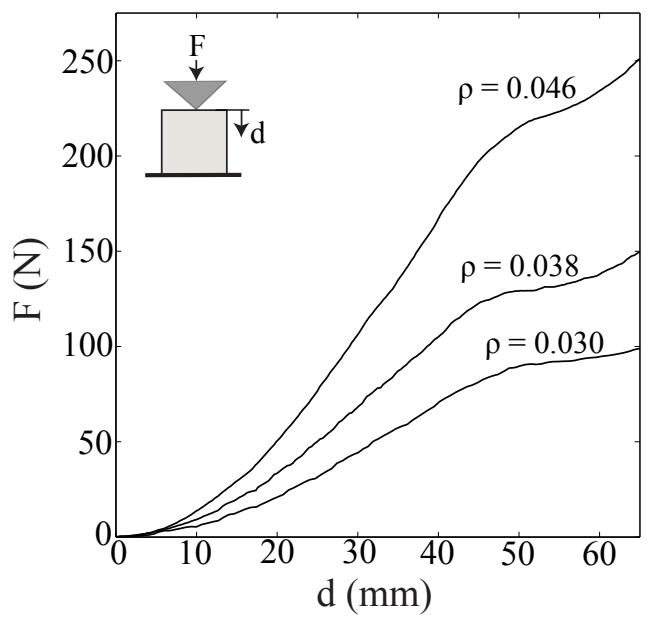

(a)

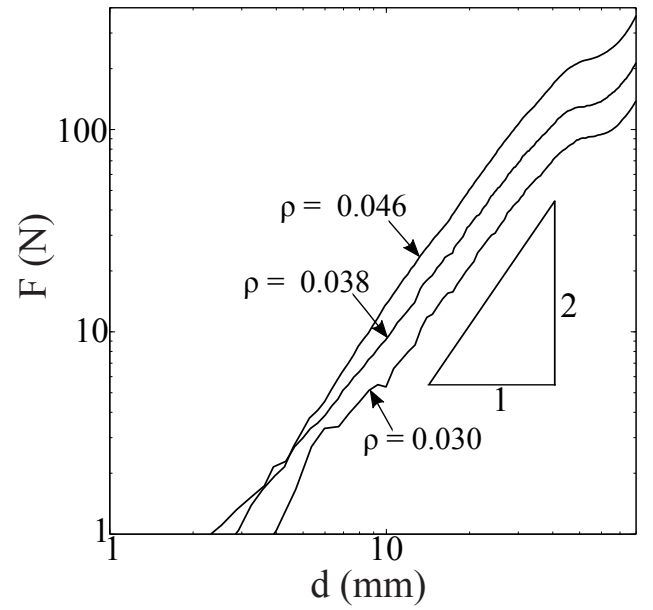

(c)

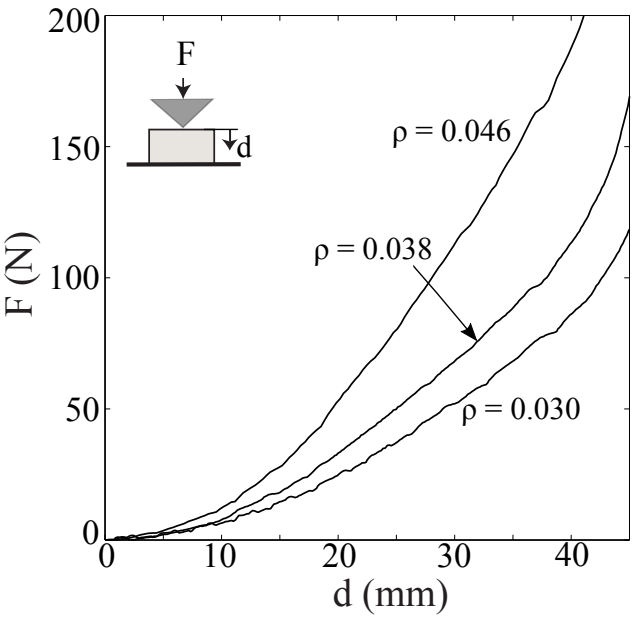

(b)

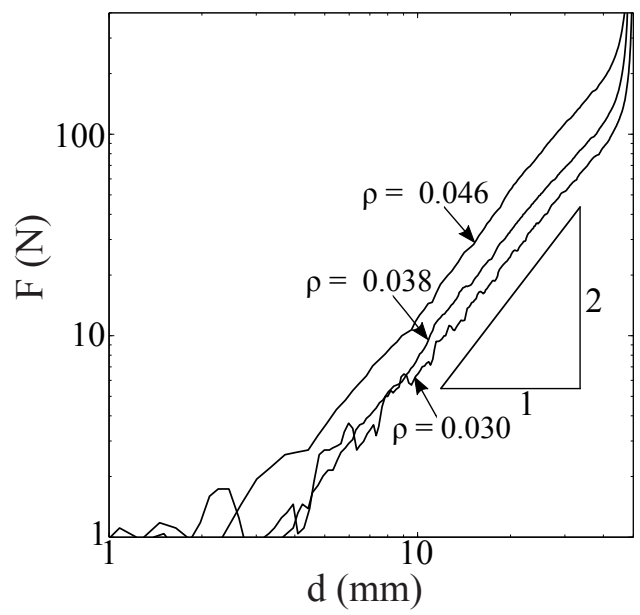

(d)

Figure 2: Force-penetration curves from experiments with the conical punch (angle of attack $=90^{\circ}$ ). $\rho$ denotes the relative density of a foam. (a) Tall specimens. Inset: schematic of the experiments. (b) Short specimens. Inset: schematic of the experiments. (c) The same as in panel a but in log-log scale. (d) The same as in panel b but in $\log -\log$ scale. Note that the force-penetration curves remain quadratic up to $d \approx 40 \mathrm{~mm}$, at which point there is either an abrupt falloff in stiffness (for the tall specimens) or an abrupt rise in stiffness (for the short specimens). 


\section{Analysis of the experiments}

The present analysis entails considerable simplifications. Nevertheless, it accounts for the most salient features of the experimental force-penetration curves and yields concrete predictions that may be readily validated via computational simulations.

Consider a foam specimen which is being penetrated by the wedge-shaped punch (Fig. 3a). Close to the tip of the punch, the strain in the foam is high; far from the tip, it is low. We might expect a smooth transition between the high strain that prevails close to the tip and the low strain that prevails far from the tip. And yet, such a smooth transition might not be possible:

Configurational phases of EOC foams. In some micromechanical models of EOC foams, the cells of a foam can flip, or transition discontinuously, between two alternative configurations (Lakes et al., 1993; Gioia et al., 2001; Pampolini and Del Piero, 2008; Dai et al., 2011b; Del Piero and Pampolini, 2012), much like an umbrella in the wind flips back and forth between a concave configuration and a convex one. (An analogous phenomenon occurs in granular aggregates and has been discussed by Gioia et al. (2002).) Where the cells of the foam have not yet flipped, the foam is in the low-strain phase (denoted by $\mathbf{L}$ in Fig. $3 \mathrm{~b}$ ); where the cells have already flipped, the foam is in the high-strain phase (denoted by $\mathbf{H}$ in Fig. 3b).

In a foam specimen which is being penetrated by the wedge-shaped punch (refer back to Fig. 3a), the tip of the punch will be surrounded by the high-strain phase, which in turn will be surrounded by the low-strain phase (Fig. 3c). The strain will be discontinuous on the interface between the phases (which we have drawn as a hemicylinder of radius $R$ in the schematic of Fig. 3c), and the stress there will equal the Maxwell stress of the foam, $\sigma_{\mathrm{M}}$ - the only stress under which the low-strain phase and the high-strain phase can coexist in stable mechanical equilibrium with one another. The Maxwell stress (as well as the attendant strains - that of the low-strain phase, $\varepsilon_{\mathrm{L}}$, and that of the high-strain phase, $\varepsilon_{\mathrm{H}}$ ) can be calculated from a knowledge of the strain-energy-density function of the foam, by imposing the Erdmann equilibrium conditions (Gioia et al., 2001; Ericksen, 1988). Thus, $\sigma_{\mathrm{M}}, \varepsilon_{\mathrm{L}}$, and $\varepsilon_{\mathrm{H}}$ are properties of the foam, and the stress on the interface is independent of the radius of the interface, $R$. It follows from equilibrium that the punching force is directly proportional to the radius of the interface, $F \propto \sigma_{\mathrm{M}} b R$, where $b$ is the depth of the specimen (measured in the direction normal to the plane of the schematic of Fig. 3c).

The similarity regime. We argue that, for as long as the interface remains distant from both the lateral boundary of the specimen and the supporting plate (Fig. 3c), there obtains a similarity regime in which $R$ (the radius of the interface) and $d$ (the penetration of the punch) are the only prevailing lengthscales (recall that the punch lacks an intrinsic lengthscale), so that $R=s d$, where $s$ is a similarity factor that depends on the relative density of the foam, $\rho$ (Appendix B). Thus, in the similarity regime, the penetration of the punch is proportional to the radius of the interface (Fig. 3d), and the constant of proportionality (i.e., the similarity factor) depends on $\rho$. 


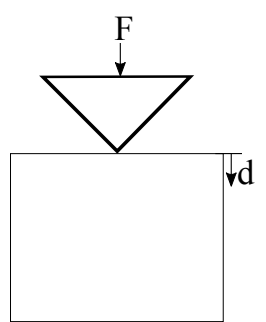

(a)

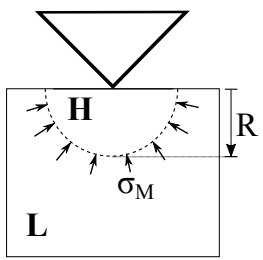

(c)

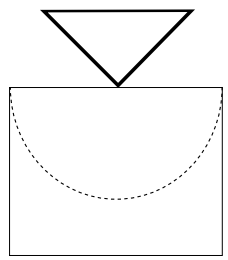

(e)

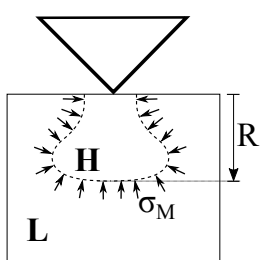

(g)

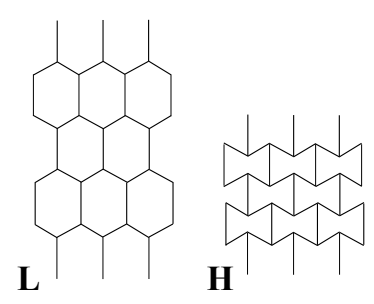

(b)

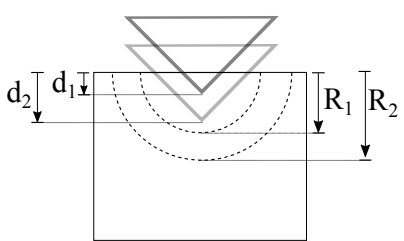

(d)

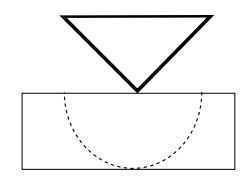

(f)

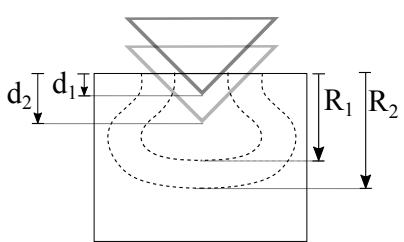

(h)

Figure 3: Analysis of an experiment with the wedge-shaped punch. (a) Schematic of the experiment. The schematic is drawn in the undeformed geometry. (b) Configurational phases of EOC foams. Schematic of the low-strain phase $\mathbf{L}$ and schematic of the high-strain phase $\mathbf{H}$ for a patch of foam consisting of 8 cells. (c) Distribution of phases in the first part of an experiment with the wedge-shaped punch. There is a strain discontinuity at the interface between the phases; the stress on the interface must be the Maxwell stress $\sigma_{\mathrm{M}}$, a property of the foam independent of the radius of the interface, $R$. (d) Illustration of the similarity regime; the radius of the interface between the phases, $R$, is proportional to the penetration of the punch, $d$. (e) The end of the similarity regime for a tall specimen. (f) The end of the similarity regime for a short specimen. (g) Variant of panel c: the equations of the analysis remain valid if the interface is a cylinder of arbitrary cross section and suitable symmetry. (h) Variant of panel d. The dimensions of the interface are set by $R$ but the shape of the interface does not change; in the similarity regime, $R \propto d$. 
By substituting in the equilibrium equation, we conclude that

$$
F \propto \sigma_{\mathrm{M}} s b d
$$

in the similarity regime, where both the Maxwell stress $\sigma_{\mathrm{M}}$ and the similarity factor $s$ depend on $\rho$. Thus, for any given value of $\rho$, the punching force is proportional to the penetration of the punch, consistent with the mechanical response observed in the first part of the experiments with the wedge-shaped punch (Fig. 1).

The same reasonings, applied to an experiment with the conical punch (where the interface may be conceived as a hemisphere of radius $R$ ), yield $R=s d$ and

$$
F \propto \sigma_{\mathrm{M}} s^{2} d^{2}
$$

for the similarity regime, where both the Maxwell stress $\sigma_{M}$ and the similarity factor $s$ depend on $\rho$. Thus, for any given value of $\rho$, the punching force is proportional to the square of the penetration of the punch, consistent with the mechanical response observed in the first part of the experiments with the conical punch (Fig. 2).

Beyond the similarity regime. Eventually, the interface reaches either the lateral boundary of the specimen or the supporting plate. For the tall specimens, the soft, stress-free lateral boundary is reached first (Fig. 3e), and the stiffness must drop. This prediction is consistent with the experimental results for the tall specimens (Fig. 1a, Fig. 2a).

For the short specimens, the supporting plate is reached first (Fig. 3f), and the stiffness must shoot up. This prediction is consistent with the experimental results for the short specimens (Fig. 1b, Fig. 2b).

Closing remarks on the analysis. We have conceived the interface between the phases as a hemicylinder of radius $R$ (wedge-shaped punch) or a hemisphere of radius $R$ (conical punch), but this is not of the essence. For the experiment with the wedge-shaped punch, for example, the interface might be a cylinder of arbitrary cross section (provided that the cross section be symmetric with respect to the axis of the punching force); thus, we can replace Fig. 3g and Fig. 3h for Fig. 3c and Fig. 3d, respectively, and the equations of the analysis will remain valid.

The principal simplification of the analysis concerns the Maxwell stress $\sigma_{\mathrm{M}}$, which we have treated as a scalar pressure, as if the foam were a fluid. To apply the equations of the analysis to the computational simulations (to which we turn next), we shall identify $\sigma_{\mathrm{M}}$ with the normal stress that acts on the interface at the point where the interface meets the axis of the punching force.

\section{Experimental observation of the interface between the phases}

So far we have assumed that there is an interface between the phases. We now seek direct empirical evidence of the interface. 


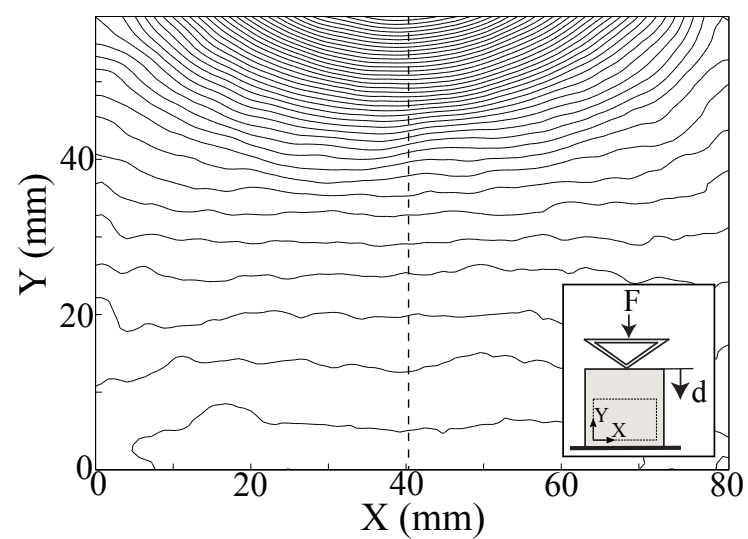

(a)

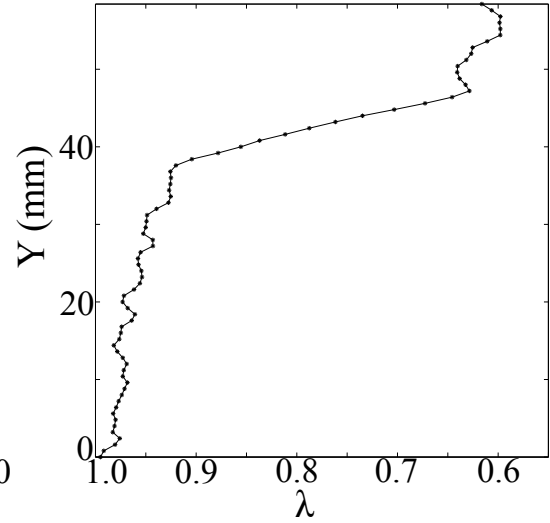

(b)

Figure 4: The interface between the phases in an experiment with the wedge-shaped punch. The relative density of the foam is 0.038; the penetration of the punch, $32.1 \mathrm{~mm}$. (a) Contour plot of the vertical component of the displacement field on a sizeable portion of one of the lateral faces of the specimen (dashed lines, inset), from digital-image-correlation (DIC) data. The vertical displacement along a contour line differs by $0.2 \mathrm{~mm}$ from the vertical displacement along each of the two adjacent contour lines. Closely-spaced contour lines correspond to the high-strain phase; far-apart contour lines correspond to the low-strain phase. (b) Plot of the stretch $\lambda(\lambda \equiv \partial y / \partial Y$; note that strain $=1-\lambda)$ along the transect indicated with a dotted line in panel a, above. The blunt discontinuity at $Y \approx 40 \mathrm{~mm}$ marks the position of the interface between the phases.

We use a digital-image correlation (DIC) method (Appendix A) to determine the displacement field on a sizeable portion of one of the lateral faces of the specimen (inset of Fig. 4a) at a particular instant during an experiment with the wedge-shaped punch. The vertical component of that displacement field may be plotted in the form of contour lines of constant vertical displacement (Fig. 4a). Where the contour lines are close together (in the upper part of Fig. 4a), we infer the presence of the high-strain phase; where the contour lines are widely spaced out (in the lower part of Fig. 4a), we infer the presence of the low-strain phase. In theory, the interface between the phases is a two-dimensional locus of strain (or stretch) discontinuity. From Fig. 4b it is apparent, however, that the strain discontinuity is blunt rather than sharp, and that the interface has a finite thickness of some $10 \mathrm{~mm}$ (as measured in the undeformed geometry). We ascribe the finite thickness of the interface to the fact that the foam has an intrinsic lengthscale of about $1 \mathrm{~mm}$, set by the size of the foam cells.

\section{Computational simulations}

We carry out computational simulations using the skeleton-and-bubble model of EOC foams (Sabuwala and Gioia, 2013). In this model, which has been added to the finite-element code ABAQUS (Sabuwala and Gioia, 2013), an EOC foam consists of a skeleton and a set of thin-walled bubbles (Fig. 5a). The skeleton and the thin-walled bubbles are mechanically coupled in parallel and contribute additively to the stress 


\section{(Appendix A).}

An often-overlooked relic of the foaming process, thin-walled bubbles are strikingly apparent under a scanning-electron microscope (Fig. 5b) but can be discerned under other types of microscope as well (Fig. 5c). The wall of a bubble is much thinner than a bar of the skeleton (Yasunaga et al., 1996), and most bubbles have holes, which preclude the rise of internal pressures. ${ }^{1}$ Even so, the bubbles make a signal contribution: they endow the foam with configurational phases (Sabuwala and Gioia, 2013).

To grasp how this comes about, recall the analogy of the umbrella in the wind (Section 3). The umbrella flips, or transitions discontinuously, between a concave configuration and a convex one. Remove the umbrella's canopy, however, and the umbrella (or the frame thereof) will transition smoothly from one configuration to the other. The canopy alters the mechanical response of the umbrella in the same way that a bubble alters the mechanical response of the cage-like cell that surrounds it (Sabuwala and Gioia, 2013).

The skeleton-and-bubble model has nine parameters (including three material parameters of polyether polyurethane: the elastic modulus, the Poisson's ratio, and the density), and each parameter has a clear physical significance. Eight of the parameters have been assigned values (Sabuwala and Gioia, 2013) on the basis of the experiments of Dai et al. (2011a), in which a set of polyether-polyurethane EOC foams in a broad range of relative densities was tested under five loading conditions (which did not include punching). The remaining parameter is the relative density of the foam, which remains free.

It bears emphasis that experimental results on punching, including those of Section 2, have not been used to assign values to the parameters of the model. None of the computational force-penetration curves of this paper has been fitted to the attendant experimental curve.

\subsection{Wedge-shaped punch}

We use two-dimensional plane-strain finite elements to model the foam specimens, and ABAQUS' "analytical rigid surface" (Hibbit et al., 2005) to model the wedge-shaped punch and the supporting plate (Appendix A).

The computational force-penetration curves are in good accord with the experimental ones (Fig. 6a-b). ${ }^{2}$ Note, in particular, the abrupt falloffs in stiffness (for the tall specimens) and the abrupt rises in stiffness (for the short specimens) that signal the end of the linear portions of the force-penetration curves.

Now, as per the analysis of Section 3, the linear portions of the force-penetration curves should correspond to the similarity regime and answer to Eq. 1 , which may be written in the rescaled form, $F / F_{0} \propto d / b$, where

\footnotetext{
${ }^{1}$ These holes are disregarded in the model, for simplicity, but the bubbles are not allowed to build internal pressures.

${ }^{2}$ The serrations in the computational force-penetration curves are artifacts of the discreteness of the finite-element mesh and occur only where two phases are present in the foam (see Fig. 15 of Sabuwala and Gioia (2013)). We have checked that, for each one of the meshes used in the simulations, a denser mesh reduces the amplitude of the serrations without changing the (mean) force--penetration curves.
} 


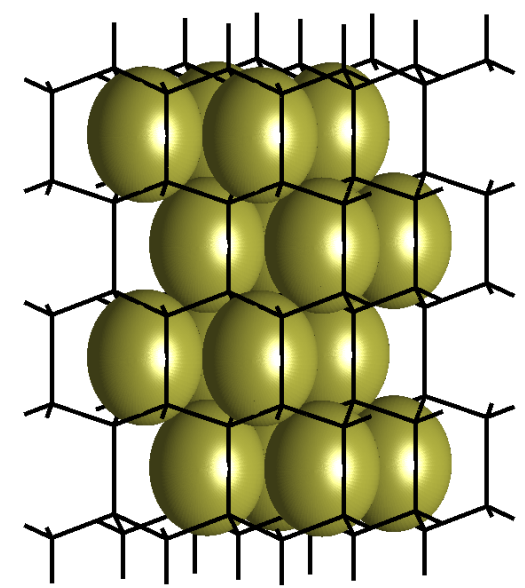

(a)

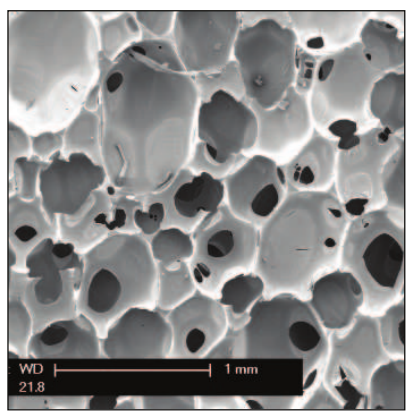

(b)

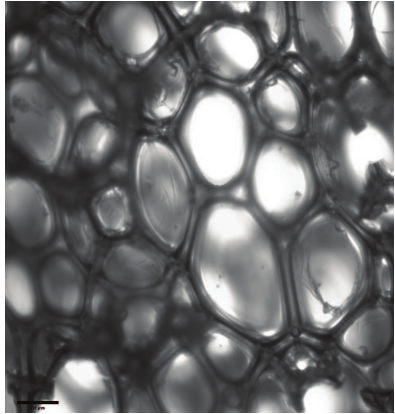

(c)

Figure 5: Skeleton-and-bubble model of EOC foams. (a) Perspective view of the skeleton and the set of thin-walled bubbles. The skeleton has hexagonal symmetry and an unambiguous rise direction (even if all the bars are of the same length). A single ellipsoidal bubble is fitted tightly within each cage-like cell of the foam. We have omitted a few bubbles to make clear the structure of the skeleton. Micrographs of a polyether-polyurethane EOC foam as seen under (b) a scanning-electron microscope and (c) a reflected-light microscope. The relative density of the foam is 0.03 . 
$F_{0} \equiv \sigma_{\mathrm{M}} s b^{2}, \sigma_{\mathrm{M}}$ is the Maxwell stress, and $s$ is the similarity factor. Thus, for consistency with the analysis of Section 3, we expect plots of $F / F_{0}$ vs. $d / b$, corresponding to different values of $\rho$, to fall on a single curve for as long as $F / F_{0}$ remains linear in $d / b$. That expectation can be confirmed in Fig. $6 c-d$, where we show 6 such plots, one for each computational simulation. (We shall presently point out the way in which we determine $\sigma_{\mathrm{M}}$ and $s$ for any given simulation.)

To visualize the interface between the phases, we plot the distribution of stretch over the domain of a tall specimen (Fig. 7a) and the distribution of stretch along the vertical axis of symmetry of the same specimen (Fig. 7b). The position of the interface is clearly marked by the stretch discontinuity in Fig. 7b.

That stretch discontinuity is blunt, and the interface has a finite thickness. This finite thickness cannot stem from the skeleton-and-bubble model of foams, which is lengthscale free (see Section 5 of Sabuwala and Gioia (2013)). But the computational simulation involves a discrete finite-element mesh, and the thickness of the interface is set by the size of the finite elements.

We identify the Maxwell stress $\sigma_{\mathrm{M}}$ with the normal stress that acts on the interface at the point where the interface meets the axis of the punching force. We have verified that that normal stress remains invariant as the penetration of the punch is increased, consistent with the notion that $\sigma_{\mathrm{M}}$ is a property of the foam.

The interface sweeps through the specimen as the penetration of the punch is increased; for as long as the the interface does not reach one of the boundaries of the specimen distal to the tip of the punch, $R$ is directly proportional to $d$ (Fig. 7c), in accord with the expected behavior in the similarity regime. To determine the similarity factor $s$, we measure the corresponding slope on the plot of $R$ vs. $d$, as indicated in Fig. 7c. (Recall that $R=s d$ in the similarity regime.)

Eventually, the interface reaches one of the boundaries of the specimen distal to the tip of the punch. For a tall specimen, the soft, stress-free lateral boundary is reached first (Fig. 8a). This event signals the breakdown of the similarity regime, and it takes place concurrently with a momentary loss of stiffness.

For a short specimen, the hard boundary in contact with the supporting plate is reached first (Fig. 8b), and the breakdown of the similarity regime takes place concurrently with a abrupt increase in stiffness.

\subsection{Conical punch}

We use three-dimensional finite elements to model one quarter of the foam specimens, and ABAQUS' "analytical rigid surface" (Hibbit et al., 2005) to model the conical punch and the supporting plate (Appendix A).

The computational force-penetration curves are in good accord with the experimental ones (Fig. 9a-d). Note, in particular, the abrupt falloffs in stiffness (for the tall specimens) and the abrupt rises in stiffness (for the short specimens) that signal the end of the quadratic portions of the force-penetration curves.

Now, as per the analysis of Section 3, the quadratic portions of the force-penetration curves should correspond to the similarity regime and answer to Eq. 2 , which may be written in the rescaled form, $F / F_{0} \propto$ 


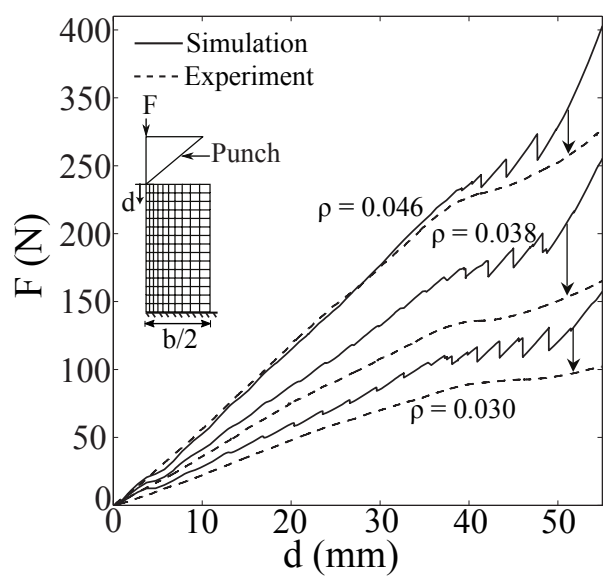

(a)

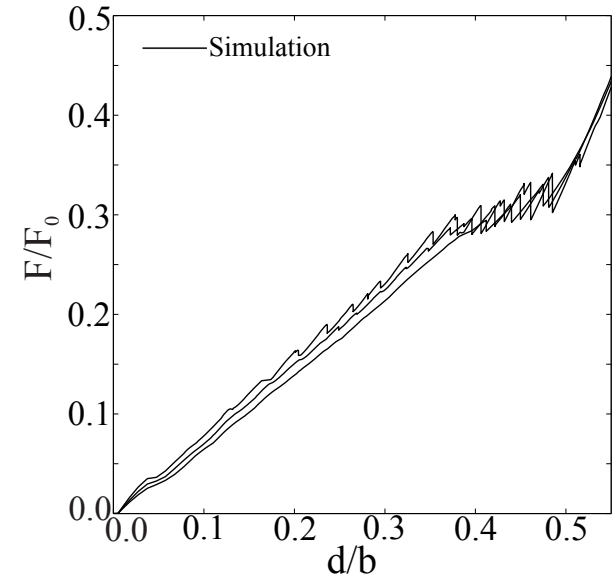

(c)

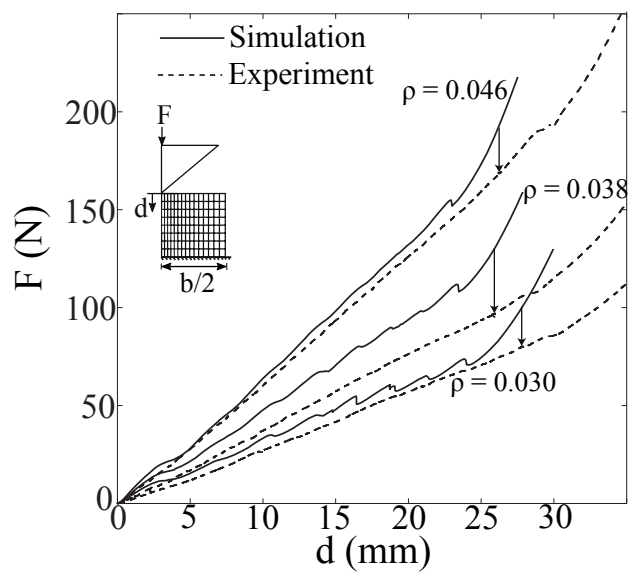

(b)

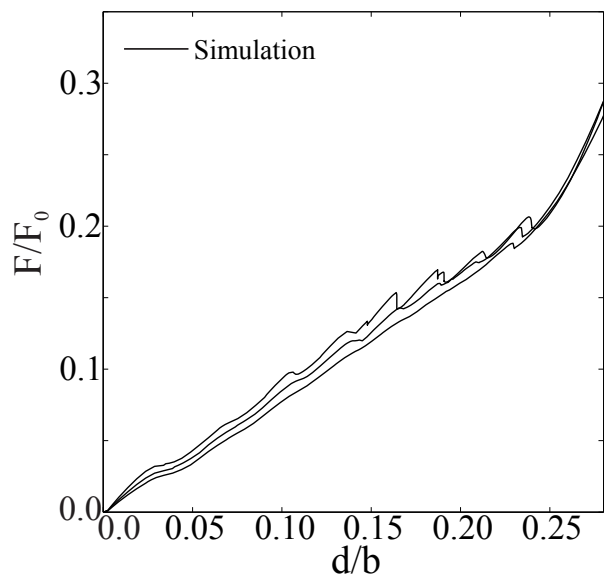

(d)

Figure 6: Force-penetration curves from experiments and computations with the wedge-shaped punch. $\rho$ denotes the relative density of the foam. (a) Tall specimens. Inset: schematic of the finite-element model. (b) Short specimens. Inset: schematic of the finite-element model. (c) The same as in panel a but with axes rescaled to verify the equation of the similarity regime, $F / F_{0} \propto d / b$. (d) The same as in panel b but with axes rescaled to verify the equation of the similarity regime, $F / F_{0} \propto d / b$. 


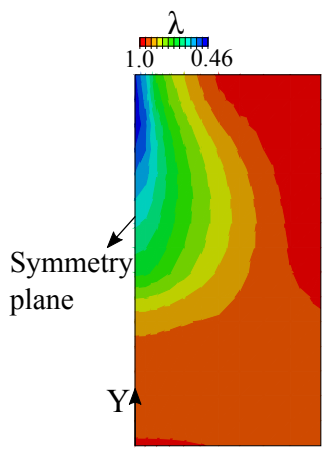

(a)

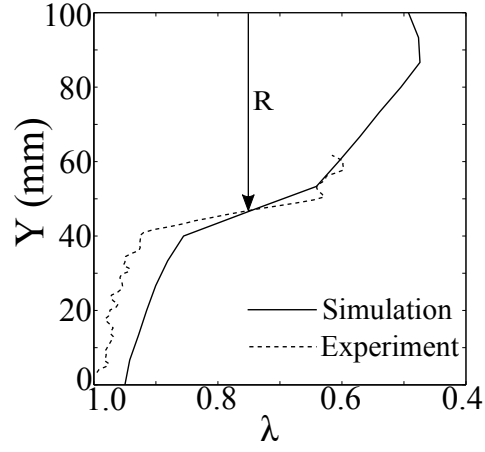

(b)

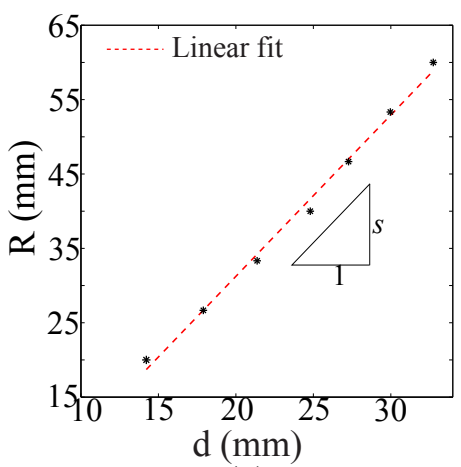

(c)

Figure 7: The interface between the phases in a computational simulation with the wedge-shaped punch. The relative density of the foam is 0.038. (a) Contour plot of the stretch $\lambda(\lambda \equiv \partial y / \partial Y)$ on the domain of the specimen for $d=31.8 \mathrm{~mm}$. (b) Plot of $\lambda$ along the vertical axis of symmetry of the same specimen and for the same penetration (solid line); also shown is the corresponding experimental plot from Fig. $4 \mathrm{~b}$ (dashed line). The discontinuity in $\lambda$ indicates the position of the interface, at a distance $R$ from the tip of the punch (cf. Fig. 3c). $R$ is measured in the undeformed geometry. (c) Plot of $R$ vs. $d$; the slope of this plot is the similarity factor $s$.

$(d / b)^{2}$, where $F_{0} \equiv \sigma_{\mathrm{M}} s^{2} b^{2}, \sigma_{\mathrm{M}}$ is the Maxwell stress, and $s$ is the similarity factor. Thus, for consistency with the analysis of Section 3, we expect plots of $F / F_{0}$ vs. $d / b$, corresponding to different values of $\rho$, to fall on a single curve for as long as $F / F_{0}$ remains quadratic in $d / b$. That expectation can be confirmed in Fig. 9e-f, where we show 6 such plots, one for each computational simulation. (We shall presently point out the way in which we determine $\sigma_{\mathrm{M}}$ and $s$ for any given simulation.)

To visualize the interface between the phases, we plot distributions of stretch along the vertical centerline of a tall specimen for 4 different penetrations (Fig. 10a). Successive positions of the interface are clearly marked by the stretch discontinuities of Fig. 10a. We identify the Maxwell stress $\sigma_{\mathrm{M}}$ with the normal stress that acts on the interface at the point where the interface meets the axis of the punching force. We have verified that that normal stress remains invariant as the penetration of the punch is increased, consistent with the notion that $\sigma_{\mathrm{M}}$ is a property of the foam.

The interface sweeps through the specimen as the penetration of the punch is increased (Fig. 10a); for as long as the the interface does not reach one of the boundaries of the specimen distal to the tip of the punch, $R$ is directly proportional to $d$ (Fig. 10b), in accord with the expected behavior in the similarity regime. To determine the similarity factor $s$, we measure the corresponding slope on the plot of $R$ vs. $d$, as indicated in Fig. 10b. (Recall that $R=s d$ in the similarity regime.)

Eventually, the interface reaches one the boundaries of the specimen distal to the tip of the punch. For a tall specimen, the soft, stress-free lateral boundary is reached first (Fig. 11a). This event signals the 


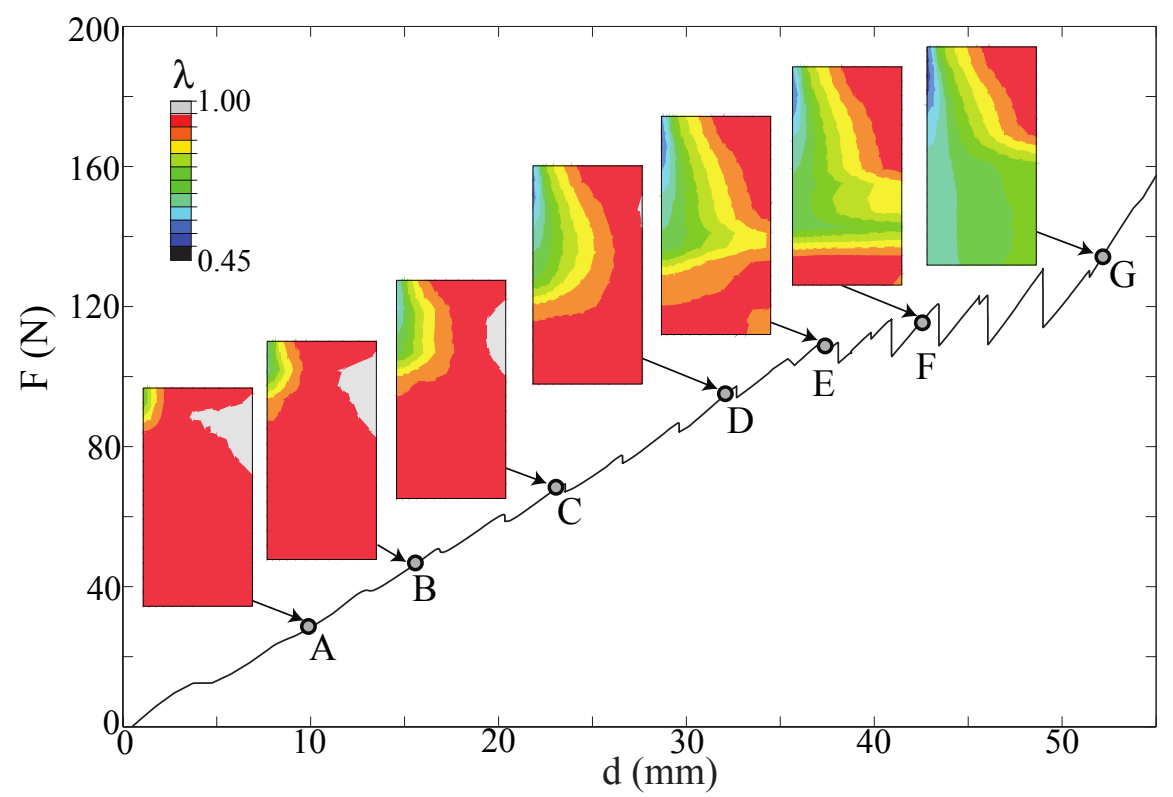

(a)

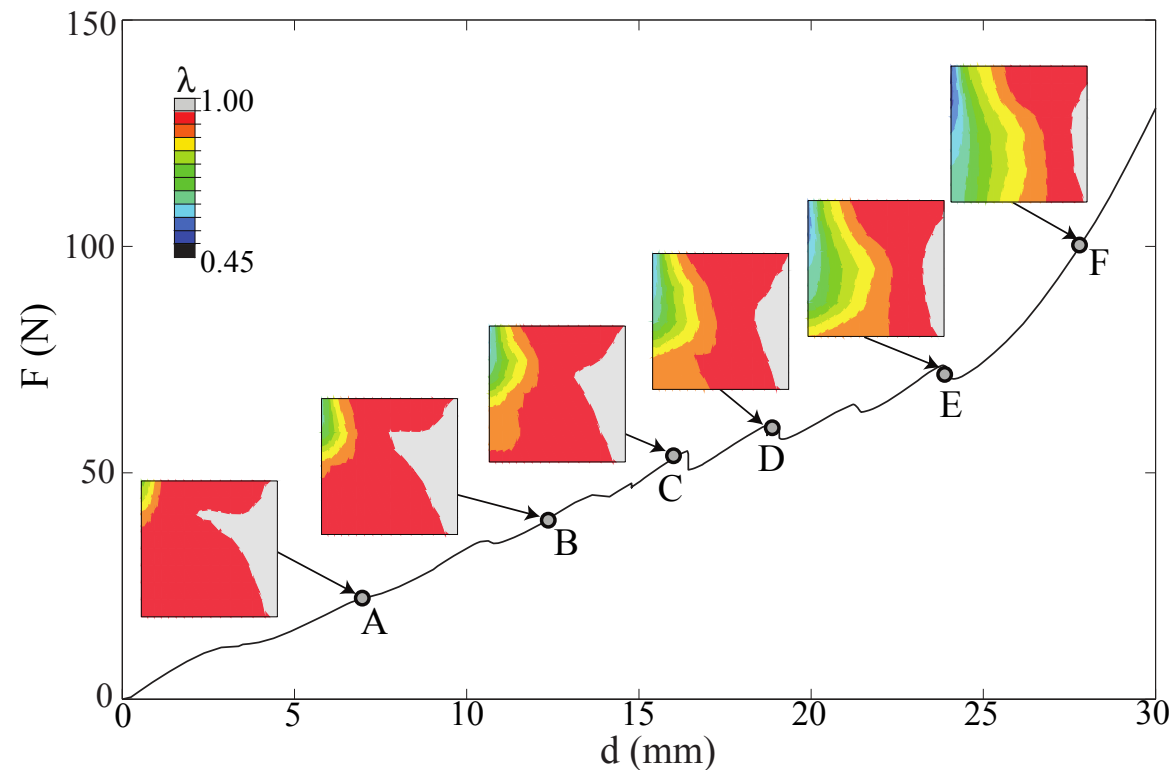

(b)

Figure 8: Computational force-penetration curves and the attendant contour plots of the stretch $\lambda$, from computational simulations with the wedge-shaped punch. The relative density of the foam is 0.03. (a) Tall specimen. Points a through $g$ correspond to $d=10.0 \mathrm{~mm}, d=16.3 \mathrm{~mm}, d=24.1 \mathrm{~mm}, d=32.2 \mathrm{~mm}, d=38.0 \mathrm{~mm}, d=41.0 \mathrm{~mm}$ and $d=51.0 \mathrm{~mm}$, respectively. b) Short specimen. Points a through $\mathrm{f}$ correspond to $d=6.90 \mathrm{~mm}, d=12.41 \mathrm{~mm}, d=16.3 \mathrm{~mm}, d=19.0 \mathrm{~mm}, d=23.9 \mathrm{~mm}$, and $d=27.8 \mathrm{~mm}$, respectively. 


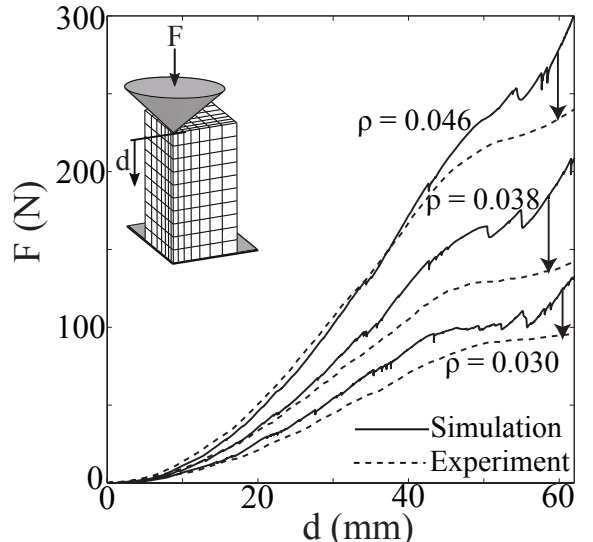

(a)

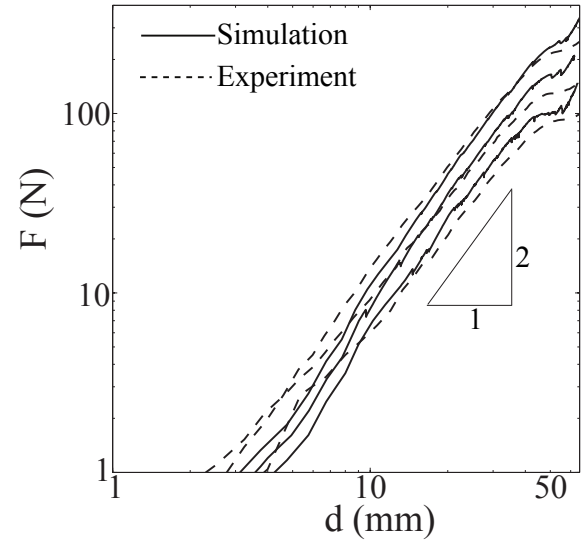

(c)

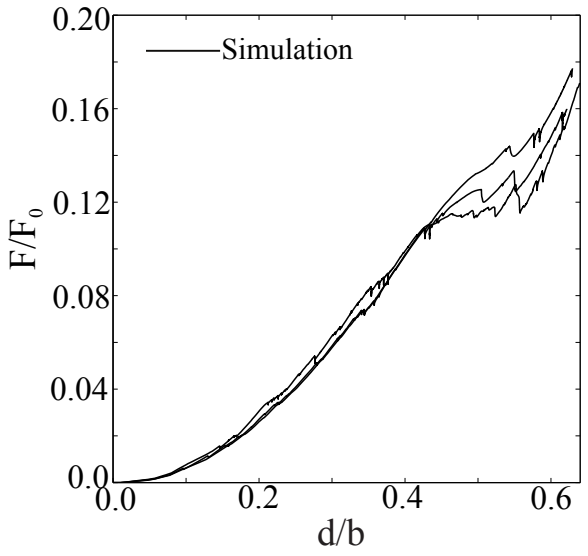

(e)

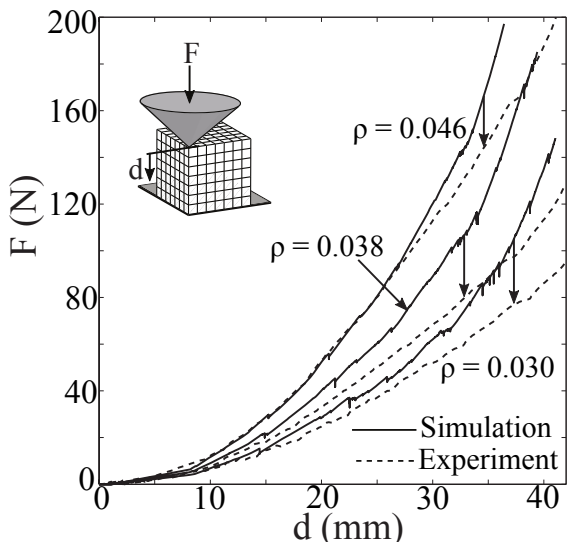

(b)

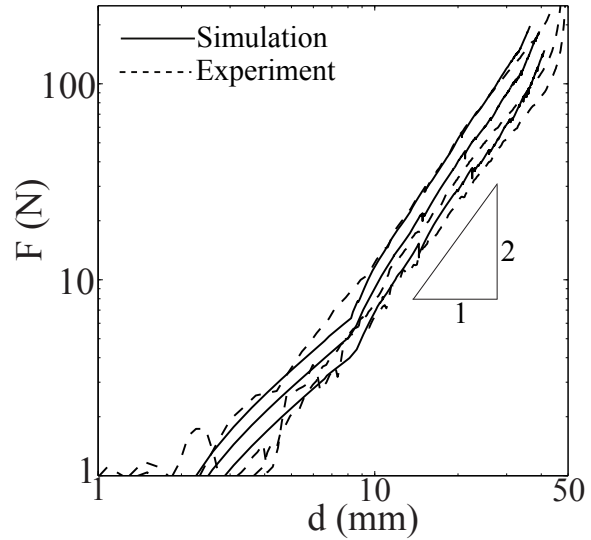

(d)

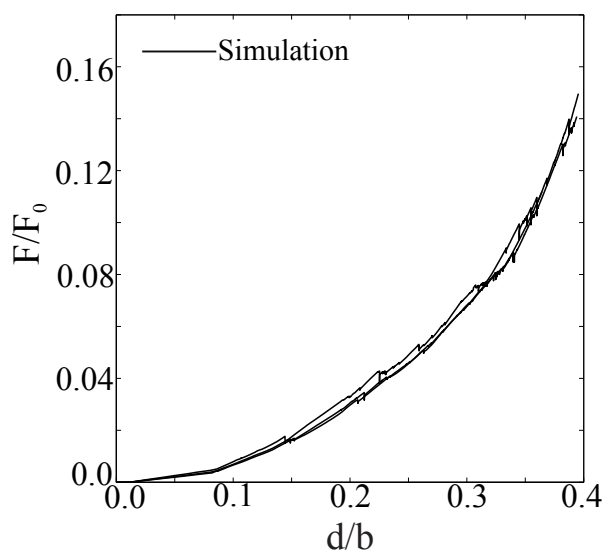

(f)

Figure 9: Force-penetration curves from experiments and computations with the conical punch. $\rho$ denotes the relative density of the foam. (a) Tall specimens. Inset: schematic of the finite-element model. (b) Short specimens. Inset: schematic of the finite-element model. (c) The same as in panel a but in log-log scale. (d) The same as in panel b but in log-log scale. (e) The same as in panel a but with axes rescaled to verify the equation of the similarity regime, $F / F_{0} \propto(d / b)^{2}$. (f) The same as in panel $\mathrm{b}$ but with axes rescaled to verify the equation of the similarity regime, $F / F_{0} \propto(d / b)^{2}$. 


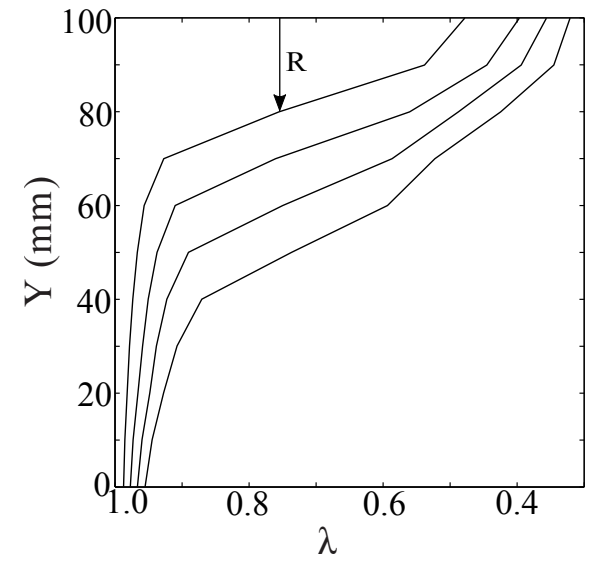

(a)

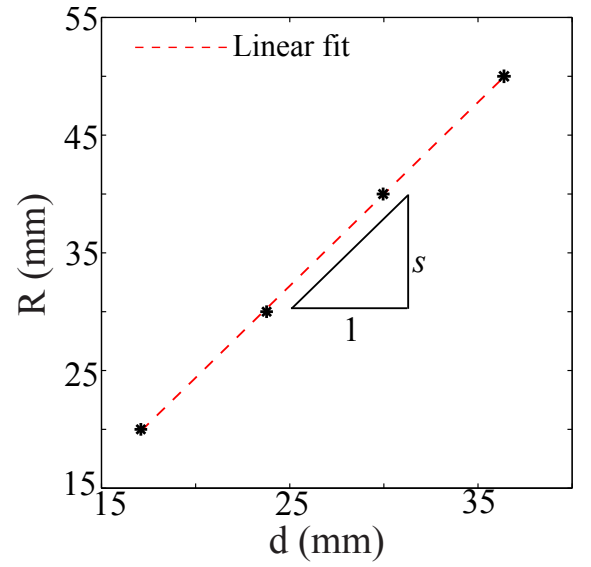

(b)

Figure 10: The interface between the phases in a computational simulation with the conical punch. The relative density of the foam is 0.03. (a) Plots of the stretch $\lambda(\lambda \equiv \partial y / \partial Y)$ along the vertical centerline of the specimen (below the tip of the punch) for $d=17.1 \mathrm{~mm}, d=23.8 \mathrm{~mm}, d=30.0 \mathrm{~mm}$, and $d=36.4 \mathrm{~mm}$. The blunt discontinuities in $\lambda$ indicate the position of the interface. (b) Plot of $R$ vs. $d$. $R$ is measured in the undeformed geometry.

breakdown of the similarity regime, and it takes place concurrently with a momentary loss of stiffness.

For a short specimen, the hard boundary in contact with the supporting plate is reached first (Fig. 11b), and the breakdown of the similarity regime takes place concurrently with a abrupt increase in stiffness.

\section{Discussion}

It has long been known that low-density elastic open-cell (EOC) foams display discontinuous strain fields under compression, even where the stress is kept uniaxial and spatially uniform throughout an experiment. Nevertheless, strain fields have scarcely been the focus of any research on EOC foams, and the cause and implications of discontinuous strain fields have remained subjects for debate.

In this paper, we have argued that EOC foams may be found in either of two distinct configurational phases. In this case, the interface between the phases is a surface of strain discontinuity, and strain fields remain discontinuous for as long as the interface is present in a foam.

To substantiate these notions, we have availed ourselves of force-penetration curves from experiments in which foam specimens of different relative densities were loaded with a wedge-shaped punch and a conical punch. By means of an analysis of the experiments (an analysis made feasible by the fact that both the wedge-shaped punch and the conical punch lack an intrinsic lengthscale), we have been able to link each salient feature of the force-penetration curves to a specific stage in the evolution of the interface between the phases. Feature by feature the links are so definite that the presence of an interface might be readily inferred from the force-penetration curves alone. Nevertheless, we have used a digital-image correlation method to 


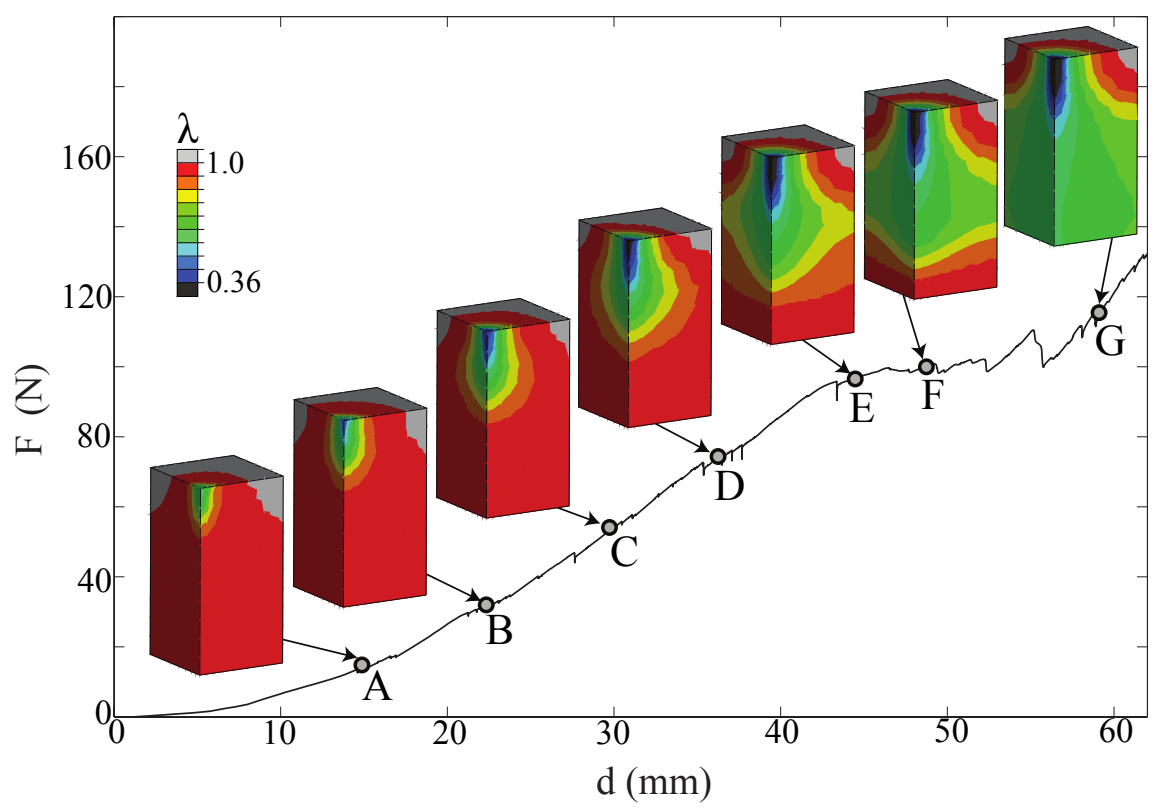

(a)

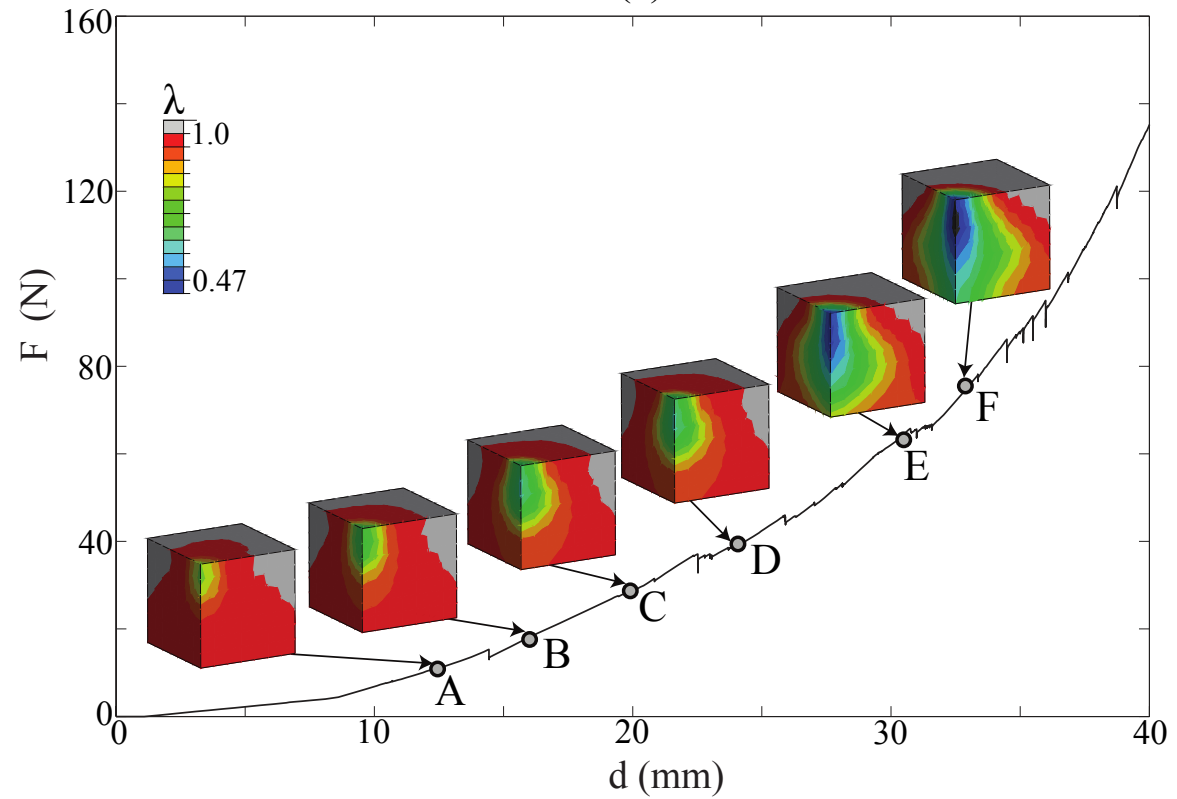

(b)

Figure 11: Force-penetration curves and the attendant contour plots of the stretch $\lambda$, from computational simulations with the conical punch. The relative density of the foam is 0.03. (a) Tall specimen. Points a through g correspond to $d=15.1 \mathrm{~mm}$, $d=22.0 \mathrm{~mm}, d=29.1 \mathrm{~mm}, d=36.1 \mathrm{~mm}, d=43.5 \mathrm{~mm}, d=48.6 \mathrm{~mm}$, and $d=57.4 \mathrm{~mm}$, respectively. (b) Short specimen. Points a through f correspond to $d=12.2 \mathrm{~mm}, d=16.0 \mathrm{~mm}, d=20.7 \mathrm{~mm}, d=24.0 \mathrm{~mm}, d=30.4 \mathrm{~mm}$, and $d=32.4 \mathrm{~mm}$ respectively. 
show that the interface was indeed present in the foam during one of the experiments with the wedge-shaped punch.

Extensive computational simulations have allowed us to put to the test a few facets of the analysis that were not readily amenable to direct experimental validation. Thus, we have been able to verify the existence of a "similarity regime" in which a shape-preserving interface expands in direct proportion to the penetration, sweeping through the specimen and substituting high-strain phase for low-strain phase to make way for the punch. On account of this similarity regime, mechanics resolves into geometry and a forcepenetration curve may be either linear or quadratic depending on whether the interface be cylindrical (as is the case for the wedge-shaped punch) or doubly curved (as is the case for the conical punch). What is more, for each type of punch, force-penetration curves corresponding to foams of differing relative densities can be made to collapse, via rescaling, on a single master curve in the similarity regime. The rescaling involves two parameters inextricably linked to the presence of configurational phases in the foams: the Maxwell stress and the similarity factor.

We have also been able to verify a prediction of the analysis to the effect that discontinuous changes in stiffness (the most salient and curious features of the experimental force-penetration curves) are but signs of the breakdown of similarity, a phenomenon whereby the interface, following extensive expansion unencumbered by boundary effects, changes shape upon being exposed to their influence. For the short specimens, the breakdown turns out to be delayed until the interface makes actual contact with the supporting plate, whereupon the force-penetration curve displays an abrupt rise in stiffness.

For the tall specimens, the breakdown occurs when a narrow band of localized deformation shoots out from the interface to emerge on the soft, stress-free lateral boundary of the specimen, a short distance way. In this case, the force-penetration curve displays an abrupt falloff in stiffness.

Our results serve to underscore the importance of configurational phases in the mechanics of EOC foams, with broad implications beyond an interest in punching. Being intrinsically discontinuous, two-phase strain fields give rise, as we have seen, to discontinuities in mechanical response which may pose computational challenges and should be factored in the interpretation of force-penetration curves, stress-strain curves and cognate forms of experimental data. On a more general note, experimental data on EOC foams remain scarce and chiefly restricted to a limited set of loading conditions. Thus, models of EOC foams have been customarily calibrated using a few experimental stress-average strain curves, mostly from uniaxial-stress tests carried out on foams of two or three relative densities, and there is a recognized need for experimental data encompassing a broader range of relative densities and a variety of loading conditions. We would like to suggest, on the basis of our findings, that future experimental research on EOC foams should include extensive measurements of strain fields. 


\section{Acknowledgments}

This research was supported financially by the Okinawa Institute of Science and Technology Graduate University, Continuum Physics Unit. The work of the second author was supported financially by the National Science Foundation under Grant CMS-0092849. We acknowledge the General Plastics Manufacturing Company of Tacoma, WA, for the donation of foam specimens.

\section{References}

Artavia, L. D., Macosko, C. W., 1994. Polyurethane flexible foam formation. In: Hilyard, N., Cunningham, A. (Eds.), Low Density Cellular Plastics. Springer Netherlands, pp. 22-55.

Barenblatt, G. I., 1996. Scaling, self-similarity, and intermediate asymptotics: dimensional analysis and intermediate asymptotics. Vol. 14. Cambridge University Press, New York.

Chan, N., Evans, K., 1998. Indentation resilience of conventional and auxetic foams. Journal of Cellular Plastics 34 (3), 231-260.

Chen, Z. C., Omidvar, M., Iskander, M., Bless, S., 2014. Modelling of projectile penetration using transparent soils. International Journal of Physical Modelling in Geotechnics 14 (3), 68-79.

Dai, X., Sabuwala, T., Gioia, G., 2011a. Experiments on elastic polyether polyurethane foams under multiaxial loading: mechanical response and strain fields. Journal of Applied Mechanics 78 (3), 031018.

Dai, X., Sabuwala, T., Gioia, G., 2011b. Lonsdaleite model of open-cell elastic foams: Theory and calibration. Journal of Elasticity 104 (1-2), 143-161.

Del Piero, G., Pampolini, G., 2012. The influence of viscosity on the response of open-cell polymeric foams in uniaxial compression: experiments and theoretical model. Continuum Mechanics and Thermodynamics $24(3), 181-199$.

Ericksen, J. L., 1988. Introduction to the Thermodynamics of Solids, 2nd Edition. Springer-Verlag, New York.

Eriksson, I., Powell, J., Kaplan, A., 2013. Melt behavior on the keyhole front during high speed laser welding. Optics and Lasers in Engineering 51 (6), 735-740.

Gioia, G., Cuitiño, A. M., Zheng, S., Uribe, T., 2002. Two-phase densification of cohesive granular aggregates. Physical Review Letters 88 (20), 204302. 
Gioia, G., Wang, Y., Cuitiño, A. M., 2001. The energetics of heterogeneous deformation in open-cell solid foams. Proceedings of the Royal Society of London A: Mathematical, Physical and Engineering Sciences 457 (2009), 1079-1096.

Hibbit, Karlsson, Sorensen, 2005. ABAQUS/Standard User's manual, version 6.5. Hibbit, Karlsson, \& Sorensen, Pawtucket, R.I.

Lakes, R., Rosakis, P., Ruina, A., 1993. Microbuckling instability in elastomeric cellular solids. Journal of Materials Science 28 (17), 4667-4672.

Lyn, G., Mills, N., 2001. Design of foam crash mats for head impact protection. Sports Engineering 4 (3), $153-163$.

Mills, N., Gilchrist, A., 2000. Modelling the indentation of low density polymer foams. Cellular Polymers 19 (6), 389-412.

Mills, N., Lyn, G., 2001. Design of foam padding for rugby posts. In: Froes, E., Haake, S. (Eds.), Materials and Science in Sports. TMS, Warrendale, PA, pp. 367-377.

Mullins, L., 1969. Softening of rubber by deformation. Rubber Chemistry and Technology 42 (1), 339-362.

Omidvar, M., Chen, Z., Iskander, M., 2014. Image-based lagrangian analysis of granular kinematics. Journal of Computing in Civil Engineering. Advance online publication. doi:10.1061/(ASCE)CP.19435487.0000433.

Pampolini, G., Del Piero, G., 2008. Strain localization in open-cell polyurethane foams: experiments and theoretical model. Journal of Mechanics of Materials and Structures 3 (5), 969-981.

Perkowitz, S., 2001. Universal Foam: Exploring the Science of Nature's Most Mysterious Substance. Anchor Books, New York.

Sabuwala, T., Gioia, G., 2013. Skeleton-and-bubble model of polyether-polyurethane elastic open-cell foams for finite element analysis at large deformations. Journal of the Mechanics and Physics of Solids 61 (3), 886-911.

Schrodt, M., Benderoth, G., Kühhorn, A., Silber, G., 2005. Hyperelastic description of polymer soft foams at finite deformations. Technische Mechanik 25 (3-4), 162-173.

Simmons, C. S., Ribeiro, A. J., Pruitt, B. L., 2013. Formation of composite polyacrylamide and silicone substrates for independent control of stiffness and strain. Lab on a Chip 13 (4), 646-649. 
Thielicke, W., Stamhuis, E. J., 2014a. Pivlab - time-resolved digital particle image velocimetry tool for matlab.

URL http://www.mathworks.com/matlabcentral/fileexchange/27659

Thielicke, W., Stamhuis, E. J., 2014b. Pivlab-towards user-friendly, affordable and accurate digital particle image velocimetry in matlab. Journal of Open Research Software 2 (1), e30.

Yasunaga, K., Neff, R., Zhang, X., Macosko, C., 1996. Study of cell opening in flexible polyurethane foam. Journal of Cellular Plastics 32 (5), 427-448.

Yu, W., Li, Y., Zheng, Y., Lim, N., Lu, M., Fan, J., 2006. Softness measurements for open-cell foam materials and human soft tissue. Measurement Science and Technology 17 (7), 1785.

\section{Appendix A. Materials and methods}

Punching experiments. Specimens are made of three polyether-polyurethane EOC foams of apparent densities 50.3, 63.0, and $77.0 \mathrm{~kg} / \mathrm{m}^{3}$. (The apparent density is the mass per unit volume of foam.) These foams are known to the manufacturer (General Plastics of Tacoma, Washington, USA) by the codes EF4003, EF-4004, and EF-4005, respectively. The polyether polyurethane of the EF series has a density of $1700 \mathrm{~kg} / \mathrm{m}^{3}$; thus, the relative densities of the three foams are $0.030,0.038$, and 0.046 .

A specimen rests on a supporting plate which is driven up at a constant rate of $3 \mathrm{~mm} / \mathrm{min}$ using a screwdriven ATS 900 Universal Testing Machine (from Applied Test Systems). The punching force is recorded by means of a $445 \mathrm{~N}$ load cell (from Interface) attached in series with the specimen, and the penetration by means of a PNL022-00 position transducer (from Spaceage Control) attached in parallel with the specimen. Where subjected to repeated loading-unloading cycles after being at rest for a few hours, polyether-polyurethane EOC foams exhibit a transient form of softening known as the Mullins effect (Mullins, 1969). The mechanical response becomes steady after three loading-unloading cycles; at least five such cycles were carried out before measuring the force-penetration curves of the paper.

Digital-image correlations. Matlab-based code PIVlab (Thielicke and Stamhuis, 2014b) is used for digitalimage correlation. This code can be freely downloaded from (Thielicke and Stamhuis, 2014a) and has been used in several studies (Simmons et al., 2013; Chen et al., 2014; Omidvar et al., 2014; Eriksson et al., 2013). The penetration of the punch is increased in steps of $1.5 \mathrm{~mm}$. A digital image of one of the lateral faces of the specimen is taken after each step, using a digital monochrome CCD camera (PULNiX TM-1300 progressive scan) fitted with a NaVITAR Zoom 700 lens. Pairs of successive images are used to determine the incremental displacement at discrete points on a sizable portion of the lateral face of the specimen (dashed lines in the inset of Fig. 4a) of size $8.2 \mathrm{~cm} \times 5.8 \mathrm{~cm}(820$ pixels $\times 580$ pixels $)$ by means of a multi-pass cross-correlation technique (available in PIVlab) that involves three increasingly smaller correlation windows of sizes $64 \times 64$ 
pixels, $32 \times 32$ pixels, and $16 \times 16$ pixels with a $50 \%$ overlap between neighbouring windows. The incremental displacements are then summed over all pairs of images to obtain the total Lagrangian displacement. The stretch is computed by numerical differentiation using the built-in Matlab function "gradient."

Computational simulations. In the skeleton-and-bubble model of EOC foams, the skeleton is assumed to work in parallel with the bubbles - just as if the skeleton, on the one hand, and the bubbles, on the other, were independently subjected to the local deformation-gradient tensor. The strain-energy density (per unit volume of foam in the undeformed geometry) is determined as the sum of a contribution from the skeleton and a contribution from the bubbles, and used as a potential to compute the first Piola-Kirchoff stress tensor at the integration point (Sabuwala and Gioia, 2013).

The frictional coefficient between the punches and the foam specimens and the frictional coefficient between the foam specimens and the supporting plates are set to the same value, 0.75 , in all computational simulations.

\section{Appendix B. The similarity regime}

Consider an experiment with the wedge-shaped punch (Fig. 3c). The governing variables are $R, d, w$ and $h$ (the width of the specimen and the height of the specimen, respectively), $\sigma_{\mathrm{M}}, \varepsilon_{\mathrm{H}}, \varepsilon_{\mathrm{L}}$, and $E_{\mathrm{H}}$ and $E_{\mathrm{L}}$ (the elastic modulus of the high-strain phase and the elastic modulus of the low-strain-phase, respectively). (The punch does not contribute a lengthscale, and we do not include the force per unit depth, $F / b$, because $F / b \propto \sigma_{M} R$ by equilibrium.) From the $\Pi$-theorem of dimensional analysis (Barenblatt, 1996), it follows that $R=s d$, where $s$ is a function of 6 dimensionless variables $\left(w / R, h / R, \varepsilon_{\mathrm{H}}, \varepsilon_{\mathrm{L}}, \sigma_{\mathrm{M}} / E_{\mathrm{H}}, \sigma_{\mathrm{M}} / E_{\mathrm{L}}\right)$. Now, provided that the interface remains distant from both the lateral boundary of the specimen and the supporting plate (so that $w / R \ll 1$ and $h / R \ll 1$ ), and assuming complete similarity with respect to both $w / R$ and $h / R$ (Barenblatt, 1996), we can drop $w / R$ and $h / R$ from the list of dimensionless variables. In this "similarity regime," $s$ becomes a function of 4 dimensionless variables $\left(\varepsilon_{\mathrm{H}}, \varepsilon_{\mathrm{L}}, \sigma_{\mathrm{M}} / E_{\mathrm{H}}\right.$, and $\left.\sigma_{\mathrm{M}} / E_{\mathrm{L}}\right)$, each one of which is in turn a function of the relative density of the foam, $\rho$. Thus, in the similarity regime, $R=s d$, where $s$ depends on $\rho$. Note that for $R=0$ we have $d=0$; thus, we neglect any penetration of the punch that might accumulate while $R=0$ - that is, before the high-strain phase becomes present in the foam. 